\title{
WIDE BINARIES AND THE AGE OF THE GALAXY
}

\author{
T.D. OSWALT, J.A. SMITH*, M.A. WOOD \\ Florida Institute of Technology \\ Department of Physics and Space Sciences \\ Melbourne, Florida 32901 USA \\ oswalt@tycho.pss.fit.edu
}

Using $B, V, R, I$ photometric parallaxes for components of wide binaries, new bolometric corrections by Bergeron et al. (1995) and cooling curves by Wood (1995), we have determined the white dwarf luminosity function (WDLF; Oswalt et al. 1996). A minimum age of $9.5_{-0.8}^{+1.1} \mathrm{Gyr}$ is implied for the Galactic disk; arbitrarily old models are consistent at the $+2 \sigma$ level. Smith (1997) confirms these results using a much larger sample and similar methodology. Using these same new models, Wood \& Oswalt (1997) show that the WDLF of Liebert et al. (1988) yields an age of $7.5_{-0.5}^{+0.5} \mathrm{Gyr}$, more than $20 \%$ younger than their original estimate.

Our WDLF implies a firm minimum age of $\sim 11 \mathrm{Gyr}$ for the Universe. Recent revisions to the extragalactic distance scale and age determinations for old globular clusters (see Chaboyer et al. 1997) have provided independent estimates for the age of the Universe that are now within $1 \sigma$ of the minimum age implied by the WDLF.

This work was supported by NSF grants AST-901624 (TDO), AST9217988 (MAW), NASA grants NGT-51086 (JAS), NAG-53103 (MAW), and the Dudley Observatory (TDO, MAW).

\section{References}

Bergeron, P., Saumon, D., Wesemael, F. 1995, ApJ 443, 764.

Chaboyer, B., Demarque, P., Kenran P. Krauss, L. 1997, ApJ, in press.

Liebert, J., Dahn, C., Monet, D. 1988, ApJ 332, 891.

Oswalt, T., Smith, J., Wood, M. 1996, Nature (Letters) 382, 692.

Smith, J.A. 1997, Ph.D. thesis, Florida Institute of Technology.

Wood, M. 1995, in White Dwarfs, ed. Koester \& Werner, (Springer), p.41.

Wood, M., Oswalt, T. 1997 ApJ, in press (preprints: http://pss.fit.edu/astro.html).

*Now at the University of Michigan. 